\title{
Fixed Point Theorems for Generalized Kannan-Type Mappings in a New Type of Fuzzy Metric Space
}

\author{
Mi Zhou (D), ${ }^{1}$ Xiao-lan Liu $\left(\mathbb{D},{ }^{2}\right.$ and Nicolae Adrian Secelean ${ }^{3}$ \\ ${ }^{1}$ School of Science and Technology, University of Sanya, Sanya, Hainan 572000, China \\ ${ }^{2}$ College of Mathematics and Statistics, Sichuan University of Science and Engineering, Zigong, Sichuan 643000, China \\ ${ }^{3}$ Department of Mathematics and Informatics, Lucian Blaga University of Sibiu, Sibiu, Romania
}

Correspondence should be addressed to Mi Zhou; mizhou330@126.com and Xiao-lan Liu; stellalwp@163.com

Received 26 February 2020; Accepted 20 April 2020; Published 31 May 2020

Academic Editor: Frédéric Mynard

Copyright (c) $2020 \mathrm{Mi}$ Zhou et al. This is an open access article distributed under the Creative Commons Attribution License, which permits unrestricted use, distribution, and reproduction in any medium, provided the original work is properly cited.

In this paper, first, we introduce a new type of $S^{*}$-fuzzy metric space which is a generalization of fuzzy metric spaces. Second, we study the topological properties of $S^{*}$-fuzzy metric spaces. Finally, we extend Kannan-type mappings to generalized Kannan-type mappings under $\phi$-gauge functions introduced by Fang in $S^{*}$-fuzzy metric spaces and prove the existence and uniqueness of fixed point for this kind of mappings. Furthermore, we also obtain the common fixed point theorems for weak compatibility along with (E.A.) property or $\left(C L R_{g}\right)$ property. Our results extend and improve very recent theorems in the related literature.

\section{Introduction}

In 1965, Zadeh [1] introduced the concept of fuzzy sets. Since then, one of the important problems is to obtain an adequate notion of fuzzy metric spaces. In 1975, Kramosil and Michálek [2] reformulated successfully the notion probabilistic metric space, introduced by Menger in 1942, in fuzzy context. After that, George and Veeramani [3] modified the concept of fuzzy metric spaces and defined a Hausdorff topology on this fuzzy space. Another approach for fuzzy metric spaces was proposed by Kaleva and Seikkala [4], by setting the distance between two points to be a nonnegative upper semicontinuous, normal, and convex fuzzy number. In the last several decades, there has been a tremendous development and growth in fuzzy mathematics. One of the branches is to obtain several kinds of generalized fuzzy metric proposed by using related generalized metric spaces: 2-metric spaces [5, 6], D-metric spaces [7], $G$-metric spaces [8], and $D^{*}$-metric spaces [9], respectively. Very recently, Sedghi et al. [10] introduced an $S$-metric space which is a generalization of $D^{*}$-metric spaces and $G$-metric spaces and justified their work by various examples and definitions related to the topology of $S$-metric spaces. Now, there arises a natural question: "how the fuzzy metric spaces can be generalized by using the concept of $S$-metric spaces?"
In 1968, Kannan [11] introduced the Kannan-type mappings as follows.

Definition 1 (see [11]). Let $(X, d)$ be a metric space and $T$ be a mapping on $X$. We say that $T$ is a Kannan-type mapping, if there exists $0 \leq k<(1 / 2)$ such that

$$
d(T x, T y) \leq k[d(x, T x)+d(y, T y)],
$$

$\forall x, y \in X$.

This kind of mapping is very important in metric fixed point theory. It is well known that Banach's contraction mappings are continuous while Kannan-type mappings are not necessary continuous. This is a big difference between these two types of mappings. Again, it may also be noted that Banach's contraction does not characterize metric completeness. In fact, Subrahmanyam [12] proved that every metric space $X$ is complete if and only if every Kannan-type mapping has a fixed point. However, it was also pointed out that Kannan's fixed point result is not an extension of Banach contraction principle. The above shows some of the reasons why the Kannan-type mappings and their generalizations have been considered as constituting an important class of mappings in fixed point theory. 
On the other hand, an interesting class of problems in fixed point theory was addressed in recent times by use of gauge (control) functions. There are several gauge (control) functions which have been used to extend Sehgal's contraction in probabilistic metric spaces. Some examples of such applications are in [13-18]. One of such gauge functions was introduced in the setup of complete Menger probabilistic metric spaces and fuzzy metric spaces by Fang [17], where the gauge function $\phi$ satisfies the condition: for each $t>0$, there exists $r \geq t$ such that $\lim _{n \longrightarrow \infty} \phi^{n}(r)=0$, which is considered as weaker than the condition presented by Francisco et al. in [19]. It is perceived that the study of fixed points for contractions under some gauge (control) functions is an important category of problems in fixed point theory.

In this reason, in Section 2, we introduce a new type of generalized fuzzy metric spaces called $S^{*}$-fuzzy metric spaces which is a generalization of $Q$-fuzzy metric spaces and $M$-fuzzy metric spaces. We also study the topological properties and prove some interesting results related its topology and convergent sequences. By using the gauge functions introduced by Fang in $S^{*}$-fuzzy metric spaces, we introduce in Section 3 the generalized Kannan-type mappings as an extension of the concept of Kannan-type mappings and prove the existence and uniqueness of fixed point for this kind of mapping. Furthermore, we also obtain the common fixed point theorems for weak compatibility along with (E.A.) or $\left(C L R_{g}\right)$ property.

\section{2. $S^{*}$ - Fuzzy Metric Spaces}

We begin by recalling some basic definitions concerning $S$-metric spaces, fuzzy metric spaces, etc.

Definition 2 (see [10]). Let $X$ be a nonempty set. An $S$-metric on $X$ is a function $S: X^{3} \longrightarrow[0, \infty)$ that satisfies the following conditions for $\forall x, y, z, a \in X$ :

(S1) $S(x, y, z)=0 \Longleftrightarrow x=y=z$.

(S2) $S(x, y, z) \leq S(x, x, a)+S(y, y, a)+S(z, z, a)$.

The pair $(X, S)$ is called an $S$-metric space.

Remark 1 (see [10]). Every $D^{*}$-metric space is a $G$-metric space and every $G$-metric space is an $S$-metric space, but in general, the converse of these implications are not true (see [10] for more details).

Definition 3 (see [20]). A mapping $*: \Pi_{i=1}^{n}[0,1] \longrightarrow[0,1]$ is called an $n^{\text {th }}$ order $t$-norm if the following conditions are satisfied:

$$
\begin{aligned}
\text { (1) } & *(0,0, \ldots, 0)=0, *(a, 1,1, \ldots, 1)=a, \forall a \in[0,1] . \\
\text { (2) } & *\left(a_{1}, a_{2}, a_{3}, \ldots, a_{n}\right)=*\left(a_{2}, a_{1}, a_{3}, \ldots, a_{n}\right)=\cdots= \\
& *\left(a_{2}, a_{3}, a_{4}, \ldots, a_{n}, a_{1}\right) . \\
\text { (3) } & a_{i} \geq b_{i}, i=1,2,3, \ldots, n \text { implies } *\left(a_{1}, a_{2}, a_{3}, \ldots, a_{n}\right) \\
& \geq *\left(b_{1}, b_{2}, b_{3}, \ldots, b_{n}\right) . \\
\text { (4) } & *\left(*\left(a_{1}, a_{2}, a_{3}, \ldots, a_{n}\right), b_{2}, b_{3}, \ldots, b_{n}\right)=*\left(a_{1},\right. \\
& \left.*\left(a_{2}, a_{3}, \ldots, a_{n}, b_{2}\right), b_{3}, \ldots, b_{n}\right)=*\left(a_{1}, a_{2}, *\left(a_{3},\right.\right.
\end{aligned}
$$

$$
\begin{aligned}
& \left.\left.a_{4}, \ldots, a_{n}, b_{2}, b_{3}\right), b_{4}, \ldots, b_{n}\right)=*\left(a_{1}, a_{2}, a_{3}, \ldots,\right. \\
& \left.a_{n-1}, *\left(a_{n}, b_{2}, b_{3}, b_{4}, \ldots, b_{n}\right)\right) .
\end{aligned}
$$

When $n=2$, we have binary $t$-norm, which is well known as $t$-norm. Typical examples of $t$-norm are Lukasiewiczt-norm $* L(a, b)=\max \{a+b-1,0\}$, product $t$-norm $* P(a, b)=a \cdot b$, and minimum $t$-norm $* M(a, b)=\min \{a, b\}$.

Definition 4 (see [21]). Let $*$ be a $t$-norm and let $*^{n}:[0,1] \longrightarrow[0,1](n \in \mathbb{N})$ be defined as follows:

$$
\begin{aligned}
*^{1}(x) & =*(x, x), \\
*^{(n+1)}(x)=* & \left(*^{n}(x), x\right), \\
& n \in \mathbb{N}, x \in[0,1] .
\end{aligned}
$$

We say that the $t$-norm is of $H$-type, if $*$ is continuous and the sequence $\left\{*^{n}(x)\right\}_{n=1}^{\infty}$ is equicontinuous at $x=1$; i.e., for every $\varepsilon \in(0,1)$, there exists $\eta \in(0,1)$, such that if $x \in(1-\eta, 1]$, then $*^{n}(x)>1-\varepsilon, \forall n \in \mathbb{N}$. A trivial example of $t$-norm of $H$-type is $* M$.

Definition 5 (see [3]). A 3-tuple $(X, M, *)$ is said to be a fuzzy metric space if $X$ is an arbitrary nonempty set, $*$ is a continuous $t$-norm, and $M$ is a fuzzy set on $X^{2} \times(0, \infty)$ satisfying the following conditions for $\forall x, y \in X$ and $t, s>0$ :

$$
\begin{aligned}
& \text { (FM1) } M(x, y, t)>0 . \\
& \text { (FM2) } M(x, y, t)=1 \Longleftrightarrow x=y . \\
& \text { (FM3) } M(x, y, t)=M(y, x, t) . \\
& \text { (FM4) } M(x, y, t) * M(y, z, s) \leq M(x, z, t+s) .
\end{aligned}
$$

(FM5) $M(x, y, \cdot):(0, \infty) \longrightarrow[0,1]$ is a continuous function.

The pair $(M, *)$ is called a fuzzy metric on $X$. Here, $M(x, y, t)$ is considered as the degree of nearness of $x$ and $y$ with respect to $t$.

Next, we define the $S^{*}$-fuzzy metric space by using the concept of $S$-metric.

Definition 6. A triple $\left(X, S^{*}, *\right)$ is called an $S^{*}$-fuzzy metric space (denoted $S^{*}$ FMS) if $X$ is an arbitrary nonempty set, * is a continuous $3^{\text {rd }} t$-norm, and $S^{*}$ is a fuzzy set on $X^{3} \times$ $(0, \infty)$ satisfying the following conditions for $\forall x, y, z, a \in X$ and $r, s, t>0$ :

$$
\begin{aligned}
& \left(S^{*} \mathrm{FM} 1\right) S^{*}(x, y, z, t)>0 . \\
& \left(S^{*} \mathrm{FM} 2\right) S^{*}(x, y, z, t)=1 \Longleftrightarrow x=y=z . \\
& \left(S^{*} \mathrm{FM} 3\right) S^{*}(x, y, z, r+s+t) \geq^{*}\left(S^{*}(x, x, a, r), S^{*}(y, y,\right. \\
& \left.a, s), S^{*}(z, z, a, t)\right) .
\end{aligned}
$$

$\left(S^{*} \mathrm{FM} 4\right) S^{*}(x, y, z, \cdot):(0, \infty) \longrightarrow[0,1]$ is a continuous function.

Example 1. Let $X=\mathbb{R}$ be the real line and $S$ be an $S$-metric on $X$ defined by $S(x, y, z)=|y+z-2 x|+|y-z|$. Then, $(X, S)$ is an $S$-metric space. Define $*(a, b, c)=a \cdot b \cdot c$, $\forall a, b, c \in[0,1]$. Let $S^{*}$ be the function on $X^{3} \times(0, \infty)$ 
defined by $S^{*}(x, y, z, t)=t /(t+S(x, y, z)), \forall x, y, z \in X$ and $t>0$.

It is easy to check that $\left(X, S^{*}, *\right)$ is an $S^{*}$-fuzzy metric space. Moreover, it is neither $Q$-fuzzy metric space nor $M$-fuzzy metric space because $S^{*}$ is not symmetric.

Example 2. Let $X=\mathbb{R}$ and $S$ be an $S$-metric defined by $S(x, y, z)=|x-y|+|y-z|, \quad \forall x, y, z \in \mathbb{R}$. Define $*(a, b, c)=a \cdot b \cdot c, \forall a, b, c \in[0,1]$, and let $S^{*}$ be the function on $\mathbb{R}^{3} \times(0, \infty)$ defined by $S^{*}(x, y, z, t)=$ $\left[\exp ^{-(S(x, y, z) / t)}\right], \forall x, y, z \in \mathbb{R}$ and $t>0$. It is also easy to verify that $\left(X, S^{*}, *\right)$ is an $S^{*}$-fuzzy metric space.

Example 3. Let $(X, M, *)$ be a fuzzy metric space with continuous $t$-norm $*$. If we denote $S^{*}: X^{3} \times$
$(0, \infty) \longrightarrow[0,1] \quad$ by $\quad S^{*}(x, y, z, t)=\quad M(x, y, t) *$ $M(y, z, t) * M(z, x, t), \quad \forall x, y, z \in X \quad$ and $t>0$, then $\left(X, S^{*}, *\right)$ is an $S^{*}$-fuzzy metric space.

Proof

(1) It is easy to see that, for every $x, y, z \in X, t>0$, $S^{*}(x, y, z, t)>0$ and $S^{*}(x, y, z, \cdot)$ is continuous.

(2) $S^{*}(x, y, z, t)=1 \Longleftrightarrow M(x, y, t)=M(y, z, t)=$ $M(z, x, t)=1 \Longleftrightarrow x=y=z, \forall x, y, z \in X, t>0$.

(3) For all $x, y, z \in X$ and $t, r, s>0$, we have

$$
\begin{aligned}
& S^{*}(x, y, z,(t+r+s)) \\
& =M(x, y,(t+r+s)) * M(y, z,(t+r+s)) * M(z, x,(t+r+s)) \\
& \geq M\left(x, a, t+\frac{s}{2}\right) * M\left(y, a, r+\frac{s}{2}\right) * M\left(y, a, r+\frac{t}{2}\right) * M\left(z, a, s+\frac{t}{2}\right) * M\left(x, a, t+\frac{r}{2}\right) \\
& \quad * M\left(z, a, s+\frac{r}{2}\right) \\
& \geq S^{*}(x, x, a, t) * S^{*}(y, y, a, r) * S^{*}(z, z, a, s) .
\end{aligned}
$$

Therefore, the conditions of Definition 6 are satisfied, and $\left(X, S^{*}, *\right)$ is an $S^{*}$-fuzzy metric space.

Example 4. Suppose that $\left(X_{1}, S_{1}^{*}, *\right)$ and $\left(X_{2}, S_{2}^{*}, *\right)$ are two $S^{*}$-fuzzy metric spaces. The fuzzy set $D^{*}$ is defined as

$$
\begin{aligned}
& \left.D^{*}\left(\left(x_{1}, x_{2}\right)\right)\left(y_{1}, y_{2}\right),\left(z_{1}, z_{2}\right), t\right) \\
& \quad=\min \left\{S_{1}^{*}\left(x_{1}, y_{1}, z_{1}, t\right), S_{2}^{*}\left(x_{2}, y_{2}, z_{2}, t\right)\right\},
\end{aligned}
$$

$\forall\left(x_{1}, x_{2}\right),\left(y_{1}, y_{2}\right),\left(z_{1}, z_{2}\right) \in X_{1} \times X_{2}$ and $t>0$ is an $S^{*}$-fuzzy metric on $X_{1} \times X_{2}$.

Proof. It is not difficult to verify that the conditions ( $\left.S^{*} \mathrm{FM} 1\right),\left(S^{*} \mathrm{FM} 2\right)$, and $\left(S^{*} \mathrm{FM} 4\right)$ in Definition 6 hold.

It remains to prove that the condition ( $S^{*}$ FM3) holds. One has

$$
\begin{aligned}
D^{*} & \left(\left(x_{1}, x_{2}\right),\left(y_{1}, y_{2}\right),\left(z_{1}, z_{2}\right),(r+s+t)\right) \\
& =\min \left\{S_{1}^{*}\left(x_{1}, y_{1}, z_{1},(r+s+t)\right), S_{2}^{*}\left(x_{2}, y_{2}, z_{2},(r+s+t)\right)\right\} \\
& \geq \min \left\{*\left(S_{1}^{*}\left(x_{1}, x_{1}, a, r\right), S_{1}^{*}\left(y_{1}, y_{1}, a, s\right), S_{1}^{*}\left(z_{1}, z_{1}, a, t\right)\right), *\left(S_{2}^{*}\left(x_{1}, x_{1}, a, r\right), S_{2}^{*}\left(y_{1}, y_{1}, a, s\right), S_{2}^{*}\left(z_{1}, z_{1}, a, t\right)\right)\right\} \\
& \geq *\left(D^{*}\left(\left(x_{1}, x_{2}\right),\left(x_{1}, x_{2}\right),(a, a), r\right), D^{*}\left(\left(y_{1}, y_{2}\right),\left(y_{1}, y_{2}\right),(a, a), s\right), D^{*}\left(\left(z_{1}, z_{2}\right),\left(z_{1}, z_{2}\right),(a, a), t\right)\right),
\end{aligned}
$$

$\forall\left(x_{1}, x_{2}\right),\left(y_{1}, y_{2}\right),\left(z_{1}, z_{2}\right) \in X_{1} \times X_{2}$ and $t>0$, as required. Consequently, the fuzzy set $D^{*}$ is an $S^{*}$-fuzzy metric on $X_{1} \times X_{2}$.
Proposition 1. If $\left(X, S^{*}, *\right)$ is an $S^{*}$-fuzzy metric space, then $S^{*}(x, x, y, t)=S^{*}(y, y, x, t), \forall x, y \in X$ and $t>0$.

Proof. For each $\varepsilon>0$, from ( $S^{*}$ FM3), we deduce 


$$
\begin{aligned}
& S^{*}(x, x, y, 2 \varepsilon+t) \geq *\left(S^{*}(x, x, x, \varepsilon), S^{*}(x, x, x, \varepsilon), S^{*}(y, y, x, t)\right)=S^{*}(y, y, x, t), \\
& S^{*}(y, y, x, 2 \varepsilon+t) \geq *\left(S^{*}(y, y, y, \varepsilon), S^{*}(y, y, y, \varepsilon), S^{*}(x, x, y, t)\right)=S^{*}(x, x, y, t) .
\end{aligned}
$$

By taking the limit in above equalities as $\varepsilon \longrightarrow 0$, we obtain $S^{*}(x, x, y, t)=S^{*}(y, y, x, t)$.

Proposition 2. $S^{*}(x, x, y, t)$ is nondecreasing with respect to $t, \forall x, y \in X$.

Proof. Suppose that $S^{*}(x, x, y, t)>S^{*}(x, x, y, s)$ for some $0<t<s$, with $(s / 2)>t$.

Then,

$$
\begin{aligned}
& *\left(S^{*}\left(x, x, x, \frac{s}{2}\right), S^{*}\left(x, x, x, \frac{s}{2}-t\right), S^{*}(y, y, x, t)\right) \\
& \quad \leq S^{*}(x, x, y, s)<S^{*}(x, x, y, t) .
\end{aligned}
$$

By $\left(S^{*} \mathrm{FM} 2\right)$, we have

$$
S^{*}\left(x, x, x, \frac{s}{2}\right)=S^{*}\left(x, x, x, \frac{s}{2}-t\right)=1 .
$$

Therefore, by Proposition 1 and Definition 3, we have $S^{*}(y, y, x, t)=S^{*}(x, x, y, t) \leq S^{*}(x, x, y, s)<S^{*}(x, x, y, t)$,

which is a contradiction. Hence, the conclusion holds.

Proposition 3. Let $\left(X, S^{*}, *\right)$ be an $S^{*}-$ fuzzy metric space. Then, for $\forall x, y \in X$, it follows that

(1) $S^{*}\left(x, y, y, t_{1}\right) \geq S^{*}\left(x, x, y, t_{2}\right), \forall t_{1}>t_{2}>0$.

(2) $S^{*}\left(x, y, x, t_{1}\right) \geq S^{*}\left(x, x, y, t_{2}\right), \forall t_{1}>t_{2}>0$.

Proof. The conclusion easily follows from Definition 6 and Proposition 2.

Theorem 1. Let $\left(X, S^{*}, *\right)$ be an $S^{*}$-fuzzy metric space. For every $x \in X, t>0$, the open ball $B(x, r, t)$ with center $x$ and radius $0<r<1$ is defined by

$$
B(x, r, t)=\left\{y \in X: S^{*}(y, y, x, t)>1-r\right\} .
$$

Then,

$T_{S^{*}}:=$

$\{T \subset X:[x \in T \Longleftrightarrow \exists t>0, r \in(0,1) \operatorname{such}$ that $B(x, r, t) \subseteq T]\}$ is a topology on $X$.

Proof. It is obvious that $\varnothing$ and $X$ belong to $T_{S^{*}}$.

Let $\left\{T_{i}\right\}_{i \in I} \subseteq T_{S^{*}}$ and $T=\cup_{i \in I} T_{i}$. We will show that $T \in T_{S^{*}}$.

Choose $x \in T$. There exists $i_{0} \in I$ such that $x \in T_{i_{0}}$. As $T_{i_{0}} \in T_{S^{*}}$, there exist $t>0$ and $r \in(0,1)$ such that $B(x, r, t) \subseteq T_{i_{0}}$. Thus, $B(x, r, t) \subseteq \cup_{i \in I_{n}} T_{i}=T$.

Now, let $\left\{T_{i}\right\}_{i=1}^{n} \subseteq T_{S^{*}}$ and $T=\cap T_{i}$. We will show that $T \in T_{S^{*}}$.

If $x \in T$, then $x \in T_{i}, \forall i \in\{1,2, \ldots, n\}$. Thus, one can find $t_{i}>0$ and $r_{i} \in(0,1)$ such that $B\left(x, r_{i}, t_{i}\right) \subseteq T_{i}, \forall i \in\{1,2, \ldots, n\}$.
Putting $\quad r=\min \left\{r_{1}, r_{2}, \ldots, r_{n}\right\}$ and $t=\min \left\{t_{1}, t_{2}, \ldots, t_{n}\right\}$, one obtains $B(x, r, t) \subseteq B\left(x, r_{i}, t_{i}\right), \forall i$. for all $i$ in $\{1,2, \ldots, n\}$, Indeed, for $y \in B(x, r, t)$, we have $S^{*}(y, y, x, t)>1-r \geq 1-r_{i}, \forall i$. for all $i$ in $\{1,2, \ldots, n\}$, Since, $t \leq t_{i}$, we have $S^{*}(y, y, x, t) \leq$ $S^{*}\left(y, y, x, t_{i}\right), \forall i \in\{1,2, \ldots, n\}$. Thus, $S^{*}\left(y, y, x, t_{i}\right) \geq 1-r_{i}, \forall i \in$ $\{1,2, \ldots, n\}$. Hence, $y \in B\left(x, r_{i}, t_{i}\right), \forall i \in\{1,2, \ldots, n\}$. Therefore, $B(x, r, t) \subseteq T_{i}, \forall i \in\{1,2, \ldots, n\}$, so $B(x, r, t) \subseteq \cap^{n} T_{i}=T$.

Theorem 2. Every $S^{*}-$ fuzzy metric space is Hausdorff.

Proof. Let $\left(X, S^{*}, *\right)$ be an $S^{*}$-fuzzy metric space, and let $x$ and $y$ be two distinct points of $X$. Then, $0<S^{*}(x, x, y, t)<1$.

For some $t>0$, we denote $r=S^{*}(x, x, y, t)$. For every $r_{0}$, $r<r_{0}<1$, we can find $r_{1}$ such that $r_{1} * r_{1} * r_{1} \geq r_{0}$. Now, we consider the open balls $B_{1}\left(x, 1-r_{1},(t / 3)\right)$ and $B_{2}\left(y, 1-r_{1},(t / 3)\right)$. We claim that

$$
B_{1}\left(x, 1-r_{1}, \frac{t}{3}\right) \cap B_{2}\left(y, 1-r_{1}, \frac{t}{3}\right)=\varnothing .
$$

Indeed, if there exists $z \in B_{1}\left(x, 1-r_{1},(t / 3)\right) \cap$ $B_{2}\left(y, 1-r_{1},(t / 3)\right)$, then

$$
\begin{aligned}
r & =S^{*}(x, x, y, t) \geq *\left(S^{*}\left(x, x, z, \frac{t}{3}\right), S^{*}\left(x, x, z, \frac{t}{3}\right), S^{*}\left(y, y, z, \frac{t}{3}\right)\right) \\
& >*\left(r_{1}, r_{1}, r_{1}\right) \geq r_{0}>r,
\end{aligned}
$$

which is a contradiction. Therefore, $\left(X, S^{*}, *\right)$ is Hausdorff.

Definition 7. Let $\left(X, S^{*}, *\right)$ be an $S^{*}$-fuzzy metric space and $A \subseteq X$. We say that $A$ is $F_{S^{*}}$ - bounded if, for every $x, y \in A$ and $t>0$, there exists $r \in(0,1)$ such that $S^{*}(x, x, y, t) \geq 1-r$. Furthermore, a sequence $\left\{x_{n}\right\}$ in $X$ is said to be $F_{S^{*}}$-bounded if, for $\forall t>0$ and all $n \in \mathbb{N}$, there exists $r \in(0,1)$ such that $x_{n} \in \bar{B}(x, r, t)$, where $\bar{B}(x, r, t)$ is a closed ball with center $x$ and radius $0<r<1$ defined by $\bar{B}(x, r, t)=\left\{y \in X: S^{*}(y, y, x, t) \geq 1-r\right\}$.

Definition 8. A sequence $\left\{x_{n}\right\}$ in $\left(X, S^{*}, *\right)$ is convergent to $x \in X$, if $S^{*}\left(x_{n}, x_{n}, x, t\right) \longrightarrow 1$ or $S^{*}\left(x, x, x_{n}, t\right) \longrightarrow 1$ as $n \longrightarrow \infty$ for each $t>0$. That is, for each $\varepsilon>0$ and $t>0$, there exists $n_{0} \in \mathbb{N}$ such that, for $\forall n \geq n_{0}, S^{*}\left(x_{n}, x_{n}, x, t\right)>1-\varepsilon$ or $S^{*}\left(x, x, x_{n}, t\right)>1-\varepsilon$. Furthermore, a subset $Y$ of an $S^{*}$-fuzzy metric space $\left(X, S^{*}, *\right)$ is said to be closed if $\left\{x_{n}\right\} \subseteq Y$ and $x_{n} \longrightarrow x$ imply $x \in Y$.

Definition 9. A sequence $\left\{x_{n}\right\}$ in an $S^{*}$-fuzzy metric space $\left(X, S^{*}, *\right)$ is said to be Cauchy if, for each $\varepsilon>0$ and $t>0$, there exists $n_{0} \in \mathbb{N}$ such that $S^{*}\left(x_{n}, x_{n}, x_{m}, t\right)>1-\varepsilon$ or $S^{*}\left(x_{m}, x_{m}, x_{n}, t\right)>1-\varepsilon, \forall m, n>n_{0}$. If every Cauchy sequence in $X$ is convergent in $X$, then $X$ is called a complete $S^{*}$-fuzzy metric space. 
Remark 2

(1) Let $\left(X, S^{*}, *\right)$ be an $S^{*}$-fuzzy metric space. A sequence $\left\{x_{n}\right\}$ is convergent to $x$ if and only if $\left\{x_{n}\right\}$ is convergent to $x$ in the topology $T_{S^{*}}$. That is,

$$
\begin{aligned}
x_{n} \underset{T_{S^{*}}}{\longrightarrow} x & \Longleftrightarrow \forall r \in(0,1), t>0, \exists n_{0} \in \mathbb{N}: x_{n} \in B(x, r, t), \quad \forall n>n_{0}, \\
& \Longleftrightarrow \forall r \in(0,1), t>0, \exists n_{0} \in \mathbb{N}: S^{*}\left(x_{n}, x_{n}, x, t\right)>1-r, \quad \forall n>n_{0}, \\
& \Longleftrightarrow \lim _{n \rightarrow \infty} S^{*}\left(x_{n}, x_{n}, x, t\right)=1, \quad \forall t>0 .
\end{aligned}
$$

(2) It is easy to prove that the $S^{*}$-fuzzy metric space $\left(X, S^{*}, *\right)$ is complete if and only if the $S$-metric space is complete where $S^{*}(x, y, z, t)=t /(t+$ $S(x, y, z)), \forall x, y, z \in X$ and $t>0$.

Lemma 1. Let $\left(X, S^{*}, *\right)$ be an $S^{*}$-fuzzy metric space, where * is a continuous $3^{\text {rd }}$ t-norm of H-type. Let $\left\{x_{n}\right\}$ be a sequence in $X$ with $x_{n} \longrightarrow x$ and $x_{n} \longrightarrow y$ as $n \longrightarrow \infty$. Then, $x=y$.

Proof. Assume that $\left\{x_{n}\right\}$ converges to $x$ and $y$. Then, $S^{*}\left(x, x, x_{n}, r\right) \underset{n}{\longrightarrow} 1$, for each $r>0$, and $S^{*}\left(y, y, x_{n}\right.$, $t-2 r) \underset{n}{\longrightarrow} 1$, for each $t-2 r>0$ :

$$
S^{*}(x, x, y, t) \geq *\left(S^{*}\left(x, x, x_{n}, r\right), S^{*}\left(x, x, x_{n}, r\right), S^{*}\left(y, y, x_{n}, t-2 r\right)\right) \underset{n}{\longrightarrow} 1 .
$$

Therefore, $x=y$.

Lemma 2. Let $\left(X, S^{*}, *\right)$ be an $S^{*}$-fuzzy metric space, where * is a continuous $3^{\text {rd }}$ t-norm of $H$-type and $\left\{x_{n}\right\}$ be a convergent sequence. Then,

(1) $\left\{x_{n}\right\}$ is $F_{S^{*}}-$ bounded and its limit is unique.

(2) $\left\{x_{n}\right\}$ is a Cauchy sequence.

(3) Every subsequence of $\left\{x_{n}\right\}$ converges to the same limit.

(4) Every Cauchy sequence is $F_{S^{*}}-$ bounded.

(5) If every Cauchy sequence $\left\{x_{n}\right\}$ in $X$ has a subsequence $\left\{x_{n}\right\}$ such that $\left\{x_{n_{k}}\right\} \longrightarrow x$, then $x_{n} \longrightarrow x$.

Proof

(1) First, we show that the convergent sequence $\left\{x_{n}\right\}$ is $F_{S^{*}}$-bounded. Suppose that $x_{n} \longrightarrow x$. Then, for each $\varepsilon \in(0,1)$ and $t>0$, there exists $n_{0} \in \mathbb{N}$ such that $S^{*}\left(x_{n}, x_{n}, x,(t / 3)\right)>1-\varepsilon, \forall n \geq n_{0}$. Choose $x_{0} \in X$ and $s \in(0,1)$ such that $S^{*}\left(x_{0}, x_{0}, x,(t / 3)\right)>1-s$ and let $k \in(0,1)$ be such that

$\min \left\{S^{*}\left(x_{n}, x_{n}, x, \frac{t}{3}\right): n=1,2,3, \ldots, n_{0}\right\}=1-k$

Then, we can find a number $r \in(0,1)$ such that

$\min \{*(1-\varepsilon, 1-\varepsilon, 1-s), *(1-k, 1-k, 1-s)\}=1-r$.

So, for $\forall n \in \mathbb{N}$, we have

$$
S^{*}\left(x_{n}, x_{n}, x, t\right) \geq *\left(S^{*}\left(x_{n}, x_{n}, x, \frac{t}{3}\right), S^{*}\left(x_{n}, x_{n}, x, \frac{t}{3}\right), S^{*}\left(x_{0}, x_{0}, x, \frac{t}{3}\right)\right) \geq 1-r \text {. }
$$

Thus, for $\forall n \in \mathbb{N},\left\{x_{n}\right\} \subseteq \bar{B}(x, r, t)$, that is, $\left\{x_{n}\right\}$ is $F_{S^{*}}$-bounded (the bar means the closure).

Now, we show that the limit of $\left\{x_{n}\right\}$ is unique. We suppose that the convergent sequence $\left\{x_{n}\right\}$ has two distinct limits $x$ and $y$. For any $\varepsilon \in(0,1)$ and $t>0$, we can find a number $r \in(0,1)$ such that $*(1-r, 1-r, 1-r)>1-\varepsilon$. Set $\varepsilon=1-S^{*}(x, x, y, t)$. From our assumption, there exist $n_{1}$ and $n_{2} \in \mathbb{N}$ such that $\quad S^{*}\left(x_{n}, x_{n}, x,(t / 3)\right)>1-r, \quad \forall n \geq n_{1}, \quad$ and $S^{*}\left(x_{n}, x_{n}, y,(t / 3)\right)>1-r, \quad \forall n \geq n_{2} . \quad$ Taking $n_{0}=\max \left\{n_{1}, n_{2}\right\}$, we have for $n \geq n_{0}$, 


$$
\begin{aligned}
S^{*}(x, x, y, t) & \geq *\left(S^{*}\left(x_{n}, x_{n}, x, \frac{t}{3}\right), S^{*}\left(x_{n}, x_{n}, x, \frac{t}{3}\right), S^{*}\left(x_{n}, x_{n}, y, \frac{t}{3}\right)\right) \\
& \geq *(1-r, 1-r, 1-r) \\
& >1-\varepsilon \\
& =S^{*}(x, x, y, t),
\end{aligned}
$$

which implies $x=y$.

(2) For each $r, t>0$ with $t-2 r>0$, one can find $n_{0} \in \mathbb{N}$ such that, for $p \in \mathbb{N}$,

$$
\begin{aligned}
S^{*}\left(x_{n}, x_{n}, x, r\right) & \longrightarrow 1, \text { for } r>0, \\
S^{*}\left(x_{n+p}, x_{n+p}, x, t-2 r\right) & \longrightarrow 1, \text { for } r, t-2 r>0, \\
S^{*}\left(x_{n}, x_{n}, x_{n+p}, t\right) & \geq *\left(S^{*}\left(x_{n}, x_{n}, x, r\right), S^{*}\left(x_{n}, x_{n}, x, r\right), S^{*}\left(x_{n+p}, x_{n+p}, x, t-2 r\right)\right) \\
& =*(1,1,1) \underset{n}{\longrightarrow} 1 .
\end{aligned}
$$

Therefore, $\left\{x_{n}\right\}$ is a Cauchy sequence.

(3) Let $\left\{x_{n_{k}}\right\}$ be a subsequence of $\left\{x_{n}\right\}$. If $x_{n} \longrightarrow x$, then for each $\varepsilon \in(0,1)$ and $t>0$, there exists $n_{0} \in \mathbb{N}$ such that $S^{*}\left(x_{n}, x_{n}, x, t\right)>1-\varepsilon, \forall n \geq n_{0}$. If $k \geq n_{0}$, then $n_{0} \leq k \leq n_{k}$ and so $S^{*}\left(x_{n_{k}}, x_{n_{k}}, x, t\right)>1-\varepsilon$ which implies that $\left\{x_{n_{k}}\right\}$ converges to $x$.

(4) Let $\left\{x_{n}\right\}$ be a Cauchy sequence in $X$. Then, for each $\varepsilon \in(0,1)$ and $t>0$, there exists $n_{0} \in \mathbb{N}$ such that $S^{*}\left(x_{n}, x_{n}, x_{m}, t\right)>1-\varepsilon, \forall m, n \geq n_{0}$. So, for $n \geq n_{0}$, we have $S^{*}\left(x_{n}, x_{n}, x_{n_{0}}, t\right)>1-\varepsilon$. Let $r=1-\min \left\{S^{*}\left(x_{n}, x_{n}, x_{n_{0}}, t\right): n=1,2, \ldots, n_{0}-1\right\}$ and choose $s=\max \{\varepsilon, r\}$. Then, $\left\{x_{n}\right\} \subseteq \bar{B}(x, r, t)$, that is, $\left\{x_{n}\right\}$ is $F_{S^{*}}$-bounded.

(5) Let $t>0$ and $\varepsilon \in(0,1)$. We can find a number $r \in(0,1)$ such that $*(1-r, 1-r, 1-r) \geq 1-\varepsilon$. Since $\left\{x_{n}\right\}$ is a Cauchy sequence in $X$, there exists $n_{0} \in \mathbb{N}$ such that $S^{*}\left(x_{n}, x_{n}, x_{m},(t / 3)\right)>1-r$, $\forall m, n \geq n_{0}$. From $\left\{x_{n_{k}}\right\} \rightarrow x$, there exists a positive integer $i_{k}$ such ${ }^{k}$ that $i_{k}>n_{0}$ and $S^{*}\left(x_{i_{k}}, x_{i_{k}}, x,(t / 3)\right)>1-r$. For $\forall n \geq n_{0}$, we have

$$
\begin{aligned}
S^{*}\left(x_{n}, x_{n}, x, t\right) & \geq *\left(S^{*}\left(x_{n}, x_{n}, x_{i_{k}}, \frac{t}{3}\right), S^{*}\left(x_{n}, x_{n}, x_{i_{k}}, \frac{t}{3}\right), S^{*}\left(x_{i_{k}}, x_{i_{k}}, x, \frac{t}{3}\right)\right) \\
& >*(1-r, 1-r, 1-r) \\
& >1-\varepsilon,
\end{aligned}
$$

so $x_{n} \longrightarrow x$.

This completes the proof.

Lemma 3. Let $\left(X, S^{*}, *\right)$ be an $S^{*}$-fuzzy metric space, where $*$ is a continuous $3^{r d}$ t-norm of H-type. Let $\left\{x_{n}\right\}$ and $\left\{y_{n}\right\}$ be two sequences in $X$ with $x_{n} \underset{n}{\longrightarrow} x, y_{n} \underset{n}{\longrightarrow} y$, and
$S^{*}\left(x, x, y, t_{n}\right) \longrightarrow S^{*}(x, x, y, t) . \quad$ Then, $\quad S^{*}\left(x_{n}, x_{n}\right.$, $\left.y_{n}, t_{n}\right) \underset{n}{\longrightarrow} S^{*}(\stackrel{n}{x}, x, y, t)$.

Proof. Since $\quad x_{n} \longrightarrow x, \quad y_{n} \longrightarrow y, \quad$ and $S^{*}\left(x, x, y, t_{n}\right) \longrightarrow S^{*}(x, x, y, t)$ as $n \longrightarrow \infty$, there exists $n_{0} \in \mathbb{N}$ such that $\left|t-t_{n}\right|<\delta$ for $n \geq n_{0}$ and $\delta<(t / 2)$. 
By the nondecreasing property of $S^{*}(x, x, y, t)$ with respect to $t$, we have

$$
\begin{aligned}
S^{*} & \left.x_{n}, x_{n}, y_{n}, t_{n}\right) \\
& \geq S^{*}\left(x_{n}, x_{n}, y_{n}, t-\delta\right) \\
& \geq *\left(S^{*}\left(x_{n}, x_{n}, x, \frac{\delta}{3}\right), S^{*}\left(x_{n}, x_{n}, x, \frac{\delta}{3}\right), S^{*}\left(y_{n}, y_{n}, x, t-\frac{5 \delta}{3}\right)\right) \\
& \geq *\left(S^{*}\left(x_{n}, x_{n}, x, \frac{\delta}{3}\right), S^{*}\left(x_{n}, x_{n}, x, \frac{\delta}{3}\right), *\left(S^{*}\left(y_{n}, y_{n}, y, \frac{\delta}{6}\right), S^{*}\left(y_{n}, y_{n}, y, \frac{\delta}{6}\right), S^{*}(y, y, x, t-2 \delta)\right)\right), \\
& S^{*}(x, x, y, t+2 \delta) \\
\geq & S^{*}\left(x, x, y, t_{n}+\delta\right) \\
\geq *\left(S^{*}\left(x, x, x_{n}, \frac{\delta}{3}\right), S^{*}\left(x, x, x_{n}, \frac{\delta}{3}\right), S^{*}\left(y, y, x_{n}, t_{n}+\frac{\delta}{3}\right)\right) & \geq *\left(S^{*}\left(x, x, x_{n}, \frac{\delta}{3}\right), S^{*}\left(x, x, x_{n}, \frac{\delta}{3}\right), *\left(S^{*}\left(y, y, y_{n}, \frac{\delta}{6}\right), S^{*}\left(y, y, y_{n}, \frac{\delta}{6}\right), S^{*}\left(x_{n}, x_{n}, y_{n}, t_{n}\right)\right)\right) .
\end{aligned}
$$

Since $\delta$ is arbitrary chosen, using Definition 8 and the continuity of $S^{*}(x, x, y, t)$ with respect to $t$, for large enough $n$, we have

$S^{*}(x, x, y, t) \geq S^{*}\left(x_{n}, x_{n}, y_{n}, t_{n}\right) \geq S^{*}(y, y, x, t)=S^{*}(x, x, y, t)$.

Therefore, $\lim _{n \longrightarrow \infty} S^{*}\left(x_{n}, x_{n}, y_{n}, t_{n}\right)=S^{*}(x, x, y, t)$.

Remark 3. From Lemma 3 , we can conclude that $S^{*}$ is a continuous function on $X^{3} \times(0, \infty)$ in every $S^{*}$-fuzzy metric space $\left(X, S^{*}, *\right)$.
Lemma 4. Let $\left(X, S^{*}, *\right)$ be an $S^{*}$-fuzzy metric space, where $*$ is a continuous $3^{\text {rd }} t$-norm of $H$-type. If there exists $k \in(0,1) \quad$ such that $S^{*}(x, x, y, k t) \geq S^{*}(x, x, y, t)$, $\forall x, y \in X, t>0$, and $\lim _{t \longrightarrow \infty} S^{*}(x, x, y, t)=1$, then $x=y$.

Proof. If there exists $k \in(0,1)$ such that $S^{*}(x, x, y, k t) \geq S^{*}(x, x, y, t), \forall x, y \in X$ and $t>0$. Then, we have

$$
S^{*}(x, x, y, t) \geq S^{*}\left(x, x, y, \frac{t}{k}\right) \geq S^{*}\left(x, x, y, \frac{t}{k^{2}}\right) \geq \cdots \geq S^{*}\left(x, x, y, \frac{t}{k^{n}}\right)
$$

for all positive integer $n$. Taking the limit as $n \longrightarrow \infty$, we have

$$
S^{*}(x, x, y, t)=1 \text {. }
$$

Hence, we have $x=y$.

Lemma 5. Let $\left(X, S^{*}, *\right)$ be an $S^{*}$-fuzzy metric. For each $r \in(0,1]$, define a function $S_{r}^{*}$ by $S_{r}^{*}(x, y, z)=$ $\inf _{t}\left\{t \geq 0: S^{*}(x, y, z, t)>1-r\right\}, \forall x, y, z \in X$. Then,

(1) $S_{r}^{*}(x, y, z)<t \Longleftrightarrow S^{*}(x, y, z, t)>1-r$.

(2) $S_{r}^{*}(x, y, z)=0, \forall r \in(0,1] \Longleftrightarrow x=y=z$.

(3) $S_{r}^{*}(x, x, y)=S_{r}^{*}(y, y, x), \forall x, y \in X$.
(4) If $*=*_{M}$, then, for every $r \in(0,1], S_{r}^{*}(x, y, z) \leq$ $S_{r}^{*}(x, x, a)+S_{r}^{*}(y, y, a)+S_{r}^{*}(z, z, a) \forall x, y, z, a \in X$.

Proof. It is not difficult to see that (1)-(3) hold. Now, we prove (4).

Let any $r \in(0,1]$ and $\varepsilon>0$. For $\forall x, y, z, a \in X$, we have

$$
\begin{aligned}
& S^{*}\left(x, x, a,\left(S_{r}^{*}(x, x, a)+\frac{\varepsilon}{3}\right)\right)>1-r, \\
& S^{*}\left(y, y, a,\left(S_{r}^{*}(y, y, a)+\frac{\varepsilon}{3}\right)\right)>1-r, \\
& S^{*}\left(z, z, a,\left(S_{r}^{*}(z, z, a)+\frac{\varepsilon}{3}\right)\right)>1-r .
\end{aligned}
$$


Hence, from ( $S^{*} \mathrm{FMS} 3$ ) and $*=*_{M}$, one obtains

$$
\begin{aligned}
& S^{*}\left(x, y, z,\left(S_{r}^{*}(x, x, a)+S_{r}^{*}(y, y, a)+S_{r}^{*}(z, z, a)+\varepsilon\right)\right) \\
& \quad \geq \min \left\{S^{*}\left(x, x, a,\left(S_{r}^{*}(x, x, a)+\frac{\varepsilon}{3}\right)\right), S^{*}\left(y, y, a,\left(S_{r}^{*}(y, y, a)+\frac{\varepsilon}{3}\right)\right), S^{*}\left(z, z, a,\left(S_{r}^{*}(z, z, a)+\frac{\varepsilon}{3}\right)\right)\right\} \\
& \quad>\min \{1-r, 1-r, 1-r\} \\
& \quad=1-r .
\end{aligned}
$$

which implies

$$
S_{r}^{*}(x, y, z) \leq S_{r}^{*}(x, x, a)+S_{r}^{*}(y, y, a)+S_{r}^{*}(z, z, a)+\varepsilon .
$$

Letting $\varepsilon \longrightarrow 0$, we have

$$
S_{r}^{*}(x, y, z) \leq S_{r}^{*}(x, x, a)+S_{r}^{*}(y, y, a)+S_{r}^{*}(z, z, a),
$$

$\forall x, y, z, a \in X$.

Definition 10. Let $\left(X, S^{*}, *\right)$ be an $S^{*}$-fuzzy metric space and $A$ be a nonempty subset of $X$. We define $S^{*}$-fuzzy diameter $D_{A}^{S^{*}}$ as

$$
D_{A}^{S^{*}}=\sup _{t>0}\left\{\inf S^{*}(x, x, y, t): x, y \in A\right\}
$$

Theorem 3. The $S^{*}$-fuzzy diameter $D_{A}^{S^{*}}$ has the following properties:

(1) $D_{A}^{S^{*}}=1 \Leftrightarrow A$ is a singleton.

(2) If $A \subseteq B$, then $D_{A}^{S^{*}} \geq D_{B}^{S^{*}}$.

(3) For any $x, y \in A, S^{*}(x, x, y, t) \geq D_{A}^{S^{*}}, \forall t>0$.

(4) If $A=\{x, y\}$, then $D_{A}^{S^{*}}=S^{*}(x, x, y, t), \forall t>0$.

Proof. The conclusions can be easily obtained from Definition 10.

\section{Fixed Point Theorems for Kannan-Type Mappings under $\phi$ - Gauge Functions}

Definition 11 (see [17]). Let $\Phi_{w}$ denote the class of all functions $\phi: \mathbb{R}^{+} \longrightarrow \mathbb{R}^{+}$satisfying the following condition: for each $t>0$, there exists $r \geq t$ such that $\lim _{n \longrightarrow \infty} \phi^{n}(r)=0$.

Lemma 6 (see [17]). If $\phi \in \Phi_{w}$, then for $\forall t>0$, there exists $r \geq t$ such that $\phi(r)<t$.

Definition 12. A map $\psi:[0,1] \times[0,1] \longrightarrow[0,1]$ is said to be a $\Psi$-function, if

(1) $\psi$ is monotonically increasing and continuous.

(2) $\psi(x, x)>x, \forall 0<x<1$.

(3) $\psi(1,1)=1, \psi(0,0)=0$.

Definition 13. Let $\left(X, S^{*}, *\right)$ be an $S^{*}$-fuzzy metric space, where $*$ is a continuous $3^{\text {rd }} t$-norm of $H$-type. A mapping $f: X \longrightarrow X$ is said to be a $\phi$-contraction if there exists a function $\phi \in \Phi_{w}$ such that

$$
S^{*}(f x, f y, f z, \phi(t)) \geq S^{*}(x, y, z, t),
$$

$\forall x, y, z \in X$ and $t>0$.

Lemma 7. Let $\left(X, S^{*}, *\right)$ be an $S^{*}-$ fuzzy metric space, where $*$ is a continuous $3^{\text {rd }}$ t-norm of $H$-type and a self-mapping $f$ on $X$ be a $\phi$-contraction. Let $x_{n+1}=f x_{n}$ be the iterative sequence generated by $x_{0} \in X, \forall n \in \mathbb{N}$. If there exists a function $\phi \in \Phi_{w}$ such that

(1) $\phi(t)>0, \forall t>0$.

(2) $S^{*}\left(x_{n}, x_{n}, x_{n+1}, \phi(t)\right) \geq S^{*}\left(x_{n-1}, x_{n-1}, x_{n}, t\right), \quad \forall n \in \mathbb{N}$ and $t>0$.

(3) $\lim _{t \longrightarrow \infty} S^{*}(x, x, y, t)=1, \forall x, y \in X$.

Then $\left\{x_{n}\right\}$ is a Cauchy sequence in $X$.

Proof. From Assumption 1, it follows that $\phi^{n}(t)>0, \forall t>0$. From Assumption 2, we obtain, by induction,

$$
S^{*}\left(x_{n}, x_{n}, x_{n+1}, \phi^{n}(t)\right) \geq S^{*}\left(x_{0}, x_{0}, x_{1}, t\right),
$$

$\forall n \in \mathbb{N}$ and $t>0$.

Now, we will prove that, for $\forall t>0$,

$$
\lim _{n \rightarrow \infty} S^{*}\left(x_{n}, x_{n}, x_{n+1}, t\right)=1 \text {. }
$$

Since $S^{*}\left(x_{0}, x_{0}, x_{1}, t\right) \longrightarrow 1$ as $t \longrightarrow \infty, \forall \varepsilon \in(0,1)$, there exists $t_{1}>0$ such that $S^{*}\left(x_{0}, x_{1}, x_{1}, t\right)>1-\varepsilon$. Since $\phi \in \Phi_{w}$, there exists $t_{2} \geq t_{1}$ such that $\lim _{n \longrightarrow \infty} \phi^{n}\left(t_{2}\right)=0$. Thus, for $\forall t>0$, there exists $n_{0} \geq 1$ such that $\phi^{n}\left(t_{2}\right)<t$, $\forall n \geq n_{0}$. Using the inequalities $t>\phi^{n}\left(t_{2}\right), t_{2} \geq t_{1}$, (31), and the monotonicity property of $S^{*}(x, y, z, \cdot)$ with respect to $t$, we have

$$
\begin{aligned}
S^{*}\left(x_{n}, x_{n}, x_{n+1}, t\right) & \geq S^{*}\left(x_{n}, x_{n}, x_{n+1}, \phi^{n}\left(t_{2}\right)\right) \\
& \geq S^{*}\left(x_{0}, x_{0}, x_{1}, t_{2}\right) \geq S^{*}\left(x_{0}, x_{0}, x_{1}, t_{1}\right)>1-\varepsilon,
\end{aligned}
$$

$\forall t>0$. Thus, (32) holds.

Since $\phi \in \Phi_{w}$, by Lemma 6, we deduce that, for any $t>0$, there exists $r \geq t$ such that $\phi(r)<t$.

Let $n \geq 1$ be given. Now, we show that for $\forall k \geq 1$,

$$
S^{*}\left(x_{n}, x_{n}, x_{n+k}, t\right) \geq *^{k}\left(S^{*}\left(x_{n}, x_{n}, x_{n+1}, \frac{t-\phi(r)}{2}\right)\right) \text {. }
$$


From (34), we obtain

$$
\begin{aligned}
& S^{*}\left(x_{n}, x_{n}, x_{n+1}, t\right) \\
& \quad \geq *\left(S^{*}\left(x_{n}, x_{n}, x_{n+1}, \frac{t-\phi(r)}{2}\right), S^{*}\left(x_{n}, x_{n}, x_{n+1}, \frac{t-\phi(r)}{2}\right), S^{*}\left(x_{n}, x_{n}, x_{n+1}, \frac{t-\phi(r)}{2}\right)\right) \\
& \quad \geq *^{1}\left(S^{*}\left(x_{n}, x_{n}, x_{n+1}, \frac{t-\phi(r)}{2}\right)\right) .
\end{aligned}
$$

Thus, (34) holds for $k=1$. Assume that (34) holds for some $k \geq 1$. Since $*$ is monotone, from ( $\left.S^{*} \mathrm{FM} 3\right)$ in Definition 6 and, then, by conditions (31) and (34), it follows that

$$
\begin{aligned}
S^{*} & \left.x_{n}, x_{n}, x_{n+k+1}, t\right) \\
& =S^{*}\left(x_{n}, x_{n}, x_{n+k+1},\left(\frac{t-\phi(r)}{2}+\frac{t-\phi(r)}{2}+\phi(r)\right)\right) \\
& \geq *\left(S^{*}\left(x_{n}, x_{n}, x_{n+1}, \frac{t-\phi(r)}{2}\right), S^{*}\left(x_{n}, x_{n}, x_{n+1}, \frac{t-\phi(r)}{2}\right), S^{*}\left(x_{n+k+1}, x_{n+k+1}, x_{n+1}, \phi(r)\right)\right) \\
& \geq *\left(S^{*}\left(x_{n}, x_{n}, x_{n+1}, \frac{t-\phi(r)}{2}\right), S^{*}\left(x_{n}, x_{n}, x_{n+1}, \frac{t-\phi(r)}{2}\right), S^{*}\left(x_{n+k}, x_{n+k}, x_{n}, r\right)\right) \\
& \geq *\left(S^{*}\left(x_{n}, x_{n}, x_{n+1}, \frac{t-\phi(r)}{2}\right), S^{*}\left(x_{n}, x_{n}, x_{n+1}, \frac{t-\phi(r)}{2}\right), S^{*}\left(x_{n+k}, x_{n+k}, x_{n}, t\right)\right) \\
& \geq *\left(S^{*}\left(x_{n}, x_{n}, x_{n+1}, \frac{t-\phi(r)}{2}\right), S^{*}\left(x_{n}, x_{n}, x_{n+1}, \frac{t-\phi(r)}{2}\right), *^{k}\left(S^{*}\left(x_{n}, x_{n}, x_{n+1}, \frac{t-\phi(r)}{2}\right)\right)\right. \\
& =*{ }^{k+1}\left(S^{*}\left(x_{n}, x_{n}, x_{n+1}, \frac{t-\phi(r)}{2}\right)\right),
\end{aligned}
$$

which completes the claim.

Next, we will show that $\left\{x_{n}\right\}$ is a Cauchy sequence in $X$, that is, $\lim _{n, m, l} \longrightarrow \infty S^{*}\left(x_{n}, x_{m}, x_{l}, t\right)=1, \forall t>0$.

Fix $t>0$ and $0<\varepsilon<1$. Since $\left\{*^{n}(t)\right\}$ is equicontinuous at $t=1$ and $*^{n}(1)=1$, there exists $\delta>0$ such that $*^{n}(s)>1-\varepsilon$ for $s \in(1-\delta, 1]$ and $n \geq 1$.

We first prove that $\lim _{n, m \rightarrow \infty} S^{*}\left(x_{n}, x_{n}, x_{m}, t\right) \in$ $(1-\delta, 1], \forall n \geq n_{0}$. Since $((t-\phi(r)) / 2)>0$, from (32), there exists $n_{0} \geq 1$ such that $S^{*}\left(x_{n}, x_{n}, x_{n+1},(t-\phi(r) / 2)\right)>1-\delta$, $\forall n \geq n_{0}$.

Hence, by (34), we have $S^{*}\left(x_{n}, x_{n}, x_{n+k}, t\right)>1-\varepsilon, \forall k \geq 1$. Thus, we have shown that, for $\forall t>0$,

$$
\lim _{n, m \longrightarrow \infty} S^{*}\left(x_{n}, x_{n}, x_{m}, t\right)=1 \text {. }
$$

By ( $S^{*}$ FM3) with $a=x_{m}$ in Definition 6 , we have that, for $\forall t>0$,

$$
\begin{aligned}
S^{*}\left(x_{n}, x_{m}, x_{l}, t\right) & \geq *\left(S^{*}\left(x_{n}, x_{n}, x_{m}, \frac{t}{3}\right), S^{*}\left(x_{m}, x_{m}, x_{m}, \frac{t}{3}\right), S^{*}\left(x_{l}, x_{l}, x_{m}, \frac{t}{3}\right)\right) \\
& =*\left(S^{*}\left(x_{n}, x_{n}, x_{m}, \frac{t}{3}\right), 1, S^{*}\left(x_{l}, x_{l}, x_{m}, \frac{t}{3}\right)\right) .
\end{aligned}
$$

From (37), it follows that, for $\forall t>0$, 


$$
\begin{aligned}
& \lim _{n, m \longrightarrow \infty} S^{*}\left(x_{n}, x_{n}, x_{m}, \frac{t}{3}\right)=1, \\
& \lim _{l, m \longrightarrow \infty} S^{*}\left(x_{l}, x_{l}, x_{m}, \frac{t}{3}\right)=1 .
\end{aligned}
$$

Thus, by using the continuity of $*$, we conclude that

$$
\begin{aligned}
\lim _{n, m \longrightarrow \infty} S^{*}\left(x_{n}, x_{m}, x_{m}, t\right) & \geq *\left(\lim _{n, m \longrightarrow \infty} S^{*}\left(x_{n}, x_{m}, x_{m}, \frac{t}{3}\right), 1, \lim _{l, m \longrightarrow \infty} S^{*}\left(x_{l}, x_{l}, x_{m}, \frac{t}{3}\right)\right) \\
& =*(1,1,1) \\
& =1 .
\end{aligned}
$$

Therefore, we have proved that, for $\forall t>0$,

$$
\lim _{n, m, l \longrightarrow \infty} S^{*}\left(x_{n}, x_{m}, x_{l}, t\right)=1 \text {. }
$$

This shows that $\left\{x_{n}\right\}$ is a Cauchy sequence in $X$.

Lemma 8. Let $\left\{x_{n}\right\}$ be an $S^{*}-$ fuzzy metric space $\left(X, S^{*}, *\right)$, where $*$ is a continuous $3^{\text {rd }} t$-norm of $H$-type. If there exists a function $\phi \in \Phi_{w}$ such that $S^{*}(x, x, y, \phi(t)) \geq S^{*}(x, x, y, t)$, $\forall t>0$, and $\lim _{t \rightarrow \infty} S^{*}(x, x, y, t)=1$, then $x=y$.

Proof. Since $S^{*}$ is monotone, it is obvious that $\phi(t)>0$, $\forall t>0$. By induction, we have $\phi^{n}(t)>0$, with $\forall t>0$ and $n \geq 1$ and also have, from the assumption, that

$$
S^{*}\left(x, x, y, \phi^{n}(t)\right) \geq S^{*}(x, x, y, t),
$$

$\forall t>0, n \geq 1$.

To prove that $x=y$, we need to verify that $S^{*}(x, x, y, t)=1$ for $\forall t>0$. On the contrary, suppose that there exists $t_{0}>0$ such that $S^{*}\left(x, x, y, t_{0}\right)<1$. Since $\lim _{t \rightarrow \infty} S^{*}(x, x, y, t)=1$, there exists $t_{1}>t_{0}$ such that

$$
S^{*}(x, x, y, t)>S^{*}\left(x, x, y, t_{0}\right)
$$

$\forall t>t_{1}$.

Since $\phi \in \Phi_{w}$, there exists $t_{2} \geq t_{1}$ such that $\lim _{n \longrightarrow \infty} \phi^{n}\left(t_{2}\right)=0$. Therefore, we can choose large enough $n_{0} \geq 1$ such that $\phi^{n_{0}}\left(t_{2}\right)<t_{0}$. By the monotonicity of $S^{*}$, using (42) and (43), we have
$S^{*}\left(x, x, y, t_{0}\right) \geq S^{*}\left(x, x, y, \phi^{n_{0}}\left(t_{2}\right)\right) \geq S^{*}\left(x, x, y, t_{2}\right)>S^{*}\left(x, x, y, t_{0}\right)$,

which is a contradiction. Therefore, $S^{*}(x, x, y, t)=1, \forall t>0$. Consequently, $x=y$.

Theorem 4. Let $\left(X, S^{*}, *\right)$ be a complete $S^{*}$-fuzzy metric space, where $*$ is a continuous $3^{\text {rd }}$-norm of H-type. If the mapping $f: X \longrightarrow X$ is a $\phi$-contraction, then $f$ has a unique fixed point $x \in X$ and, for $\forall x_{0} \in X, \lim _{n} f^{n}\left(x_{0}\right)=x$.

Proof. Let $x_{0}$ be an arbitrary point in $X$ and let us define the sequence $\left\{x_{n}\right\}$ by $x_{n+1}=f^{n} x_{0}, \forall n \geq 0$.

Since $f$ is a $\phi$-contraction, by (30), we have that, for $\forall t>0$,

$$
\begin{aligned}
S^{*}\left(x_{n}, x_{n}, x_{n+1}, \phi(t)\right) & =S^{*}\left(f x_{n-1}, f x_{n-1}, f x_{n}, \phi(t)\right) \\
& \geq S^{*}\left(x_{n-1}, x_{n-1}, x_{n}, t\right) .
\end{aligned}
$$

By Lemma 7, we conclude that $\left\{x_{n}\right\}$ is a Cauchy sequence in $X$. Since $X$ is complete, there exists $x \in X$ such that $\lim _{n \longrightarrow \infty} x_{n}=x$, that is, for $\forall t>0, \quad \lim _{n \longrightarrow \infty} S^{*}$ $\left(x_{n}, x_{n}, x, t\right)=1$.

Now, we will prove that $x$ is a fixed point of $f$.

Since $\phi \in \Phi_{w}$, by Lemma 6, we have that for $\forall t>0$, there exists $r \geq t$ such that $\phi(r)<t$. Putting $a=f x_{n}$ in ( $S^{*}$ FM3) of Definition 6, we have

$$
\begin{aligned}
S^{*}(x, x, f x, t) & \geq *\left(S^{*}\left(x, x, f x_{n}, \frac{t-\phi(r)}{2}\right), S^{*}\left(x, x, f x_{n}, \frac{t-\phi(r)}{2}\right), S^{*}\left(f x, f x, f x_{n}, \phi(r)\right)\right) \\
& \geq *\left(S^{*}\left(x, x, x_{n+1}, \frac{t-\phi(r)}{2}\right), S^{*}\left(x, x, x_{n+1}, \frac{t-\phi(r)}{2}\right), S^{*}\left(x, x, x_{n}, r\right)\right) .
\end{aligned}
$$

Since $\lim _{n \longrightarrow \infty} x_{n}=\lim _{n \longrightarrow \infty} x_{n+1}=x$ and $*$ is continuous, taking limit as $n \longrightarrow \infty$ in above inequality, one has

$$
S^{*}(f x, f x, x, t) \geq^{*}(1,1,1)=1 .
$$

So, $f x=x$, that is, $x$ is a fixed point of $f$.

Next, suppose that $y$ with $x \neq y$ is another fixed point of $f$. Then, for $\forall t>0$, we have 


$$
S^{*}(x, x, y, \phi(t))=S^{*}(f x, f x, f y, \phi(t)) \geq S^{*}(x, x, y, t),
$$

which implies, according to Lemma 8 , that $x=y$. Therefore, $f$ has a unique fixed point.

Example 5. Let $X=[0, \infty)$ and $*(a, b, c)=a \cdot b \cdot c$, $\forall a, b, c \in X$. Define the functions $S^{*}: X^{3} \times[0, \infty) \longrightarrow[0, \infty)$ by $S^{*}(x, y, z, t)=t /(t+S(x, y, z)), \quad \forall x, y, z \in X, \quad$ and $S(x, y, z)=|x-z|+|y-z|$. Then, $\left(X, S^{*}, *\right)$ is an $S^{*}$-fuzzy metric space.

Let $f: X \longrightarrow X$ be a mapping defined by $f x=x / 2$ and $\phi: \mathbb{R}^{+} \longrightarrow \mathbb{R}^{+}$be defined by

$$
\phi(t)=\left\{\begin{array}{l}
\frac{t}{2}, \quad 0 \leq t<1 \\
-\frac{t}{3}+\frac{4}{3}, \quad 1 \leq t \leq 2 ; \\
t-1, \quad 2<t<\infty .
\end{array}\right.
$$

Notice that $\phi(t) \in \Phi_{w}$ and $\phi(t) \geq(t / 2), \forall t \geq 0$.

Now, we verify that $f$ satisfies the $\phi$-contraction condition:

$$
\begin{aligned}
S^{*} & (f x, f y, f z, \phi(t)) \\
& =\frac{\phi(t)}{\phi(t)+|f x-f z|+|f y-f z|} \\
& =\frac{\phi(t)}{\phi(t)+|(x / 2)-(z / 2)|+|(y / 2)-(z / 2)|} \\
& \geq \frac{t / 2}{(t / 2)+|(x / 2)-(z / 2)|+|(y / 2)-(z / 2)|} \\
& =\frac{t}{t+|x-z|+|y-z|} \\
& =S^{*}(x, y, z, t) .
\end{aligned}
$$

This shows that $f$ is a $\phi$-contraction; then, $f$ has a fixed point in $X$. Indeed, 0 is the fixed point of $f$.

Definition 14 . Let $\left(X, S^{*}, *\right)$ be an $S^{*}$-fuzzy metric space, where $*$ is a continuous $3^{\text {rd }} t$-norm of $H$-type and assume that $\psi$ is a $\Psi$-function. A mapping $f: X \longrightarrow X$ is called generalized Kannan-type mapping under a gauge function $\phi$ if, for $\forall x, y \in X$,

$$
S^{*}(f x, f x, f y, \phi(t)) \geq \psi\left(S^{*}\left(x, x, f x, \frac{t_{1}}{a}\right), S^{*}\left(y, y, f y, \frac{t_{2}}{b}\right)\right),
$$

where $t, t_{1}, t_{2}>0$ with $t=t_{1}+t_{2}, a, b>0$ with $0<a+b<1$, $\phi \in \Phi_{w}$.

Lemma 9. Let $\left(X, S^{*}, *\right)$ be a complete $S^{*}$-fuzzy metric space, where $*$ is a continuous $3^{\text {rd }} t$-norm of $H$-type and $f$ be a generalized Kannan-type mapping under a gauge function $\phi$ on $X$. Assume that $\lim _{n \rightarrow \infty} S^{*}(x, x, y, t)=1$, $\forall x, y \in X, t>0$. If $x_{n}=f x_{n-1}$ is the iterative sequence generating by $x_{0} \in X$, then $\lim _{n \longrightarrow \infty} S^{*}\left(x_{n+1}, x_{n+1}, x_{n}, \phi(t)\right)=1$, $\forall t>0$.

Proof. Let $x_{0} \in X, x_{n}=f x_{n-1}, n \in \mathbb{N}$, and $t, t_{1}, t_{2}, a$, and $b$ be positive real numbers with $0<a+b<1$. From (51), for $t=t_{1}+t_{2}$, we have

$$
\begin{gathered}
S^{*}\left(x_{n+1}, x_{n+1}, x_{n}, \phi(t)\right)=S^{*}\left(f x_{n}, f x_{n}, f x_{n-1}, \phi(t)\right) \\
\quad \geq \psi\left(S^{*}\left(x_{n}, x_{n}, x_{n+1}, \frac{t_{1}}{a}\right), S^{*}\left(x_{n-1}, x_{n-1}, x_{n}, \frac{t_{2}}{b}\right)\right) .
\end{gathered}
$$

For $\forall t>0$, putting $t_{1}=(a t /(a+b)), t_{2}=(b t /(a+b))$ and $c=a+b$ in the above inequality, we have

$$
\begin{aligned}
& S^{*}\left(x_{n+1}, x_{n+1}, x_{n}, \phi(t)\right) \\
& \quad \geq \psi\left(S^{*}\left(x_{n}, x_{n}, x_{n+1}, \frac{t}{c}\right), S^{*}\left(x_{n-1}, x_{n-1}, x_{n}, \frac{t}{c}\right)\right), \quad n \in \mathbb{N} .
\end{aligned}
$$

Now, we claim that

$S^{*}\left(x_{n+1}, x_{n+1}, x_{n}, \frac{t}{c}\right) \geq S^{*}\left(x_{n}, x_{n}, x_{n-1}, \frac{t}{c}\right), t>0, \quad n \in \mathbb{N}$.

Suppose, to the contrary, there exists $t>0$ such that

$$
S^{*}\left(x_{n+1}, x_{n+1}, x_{n}, \frac{t}{c}\right)<S^{*}\left(x_{n}, x_{n}, x_{n-1}, \frac{t}{c}\right), \quad n \in \mathbb{N} \text {. }
$$

By property of $\psi$-function and (53), we have

$$
\begin{aligned}
& S^{*}\left(x_{n+1}, x_{n+1}, x_{n}, \phi(t)\right) \\
& \quad \geq \psi\left(S^{*}\left(x_{n+1}, x_{n+1}, x_{n}, \frac{t}{c}\right), S^{*}\left(x_{n}, x_{n}, x_{n-1}, \frac{t}{c}\right)\right) \\
& \quad \geq \psi\left(S^{*}\left(x_{n+1}, x_{n+1}, x_{n}, \frac{t}{c}\right), S^{*}\left(x_{n+1}, x_{n+1}, x_{n}, \frac{t}{c}\right)\right) \\
& \quad>S^{*}\left(x_{n+1}, x_{n+1}, x_{n}, \frac{t}{c}\right),
\end{aligned}
$$

which is a contradiction. So (53) and (54) imply the following inequality:

$$
S^{*}\left(x_{n+1}, x_{n+1}, x_{n}, \phi(t)\right) \geq S^{*}\left(x_{n}, x_{n}, x_{n-1}, \frac{t}{c}\right), \quad t>0, n \in \mathbb{N} \text {. }
$$

If we apply induction to the above inequality, we see that

$$
S^{*}\left(x_{n+1}, x_{n+1}, x_{n}, \phi(t)\right) \geq S^{*}\left(x_{1}, x_{1}, x_{0}, \frac{t}{c^{n}}\right), \quad t>0, n \in \mathbb{N} \text {. }
$$

Our additional assumption on $S^{*}$-fuzzy metric spaces implies that $\lim _{n \longrightarrow \infty} S^{*}\left(x_{1}, x_{1}, x_{0},\left(t / c^{n}\right)\right)=1$.

Thus, by taking the limit as $n \longrightarrow \infty$, we have that, for all $t>0$,

$$
\lim _{n \longrightarrow \infty} S^{*}\left(x_{n+1}, x_{n+1}, x_{n}, \phi(t)\right)=1, \quad t>0 .
$$


Theorem 5. Let $\left(X, S^{*}, *\right)$ be an $S^{*}-$ fuzzy metric space, where $*$ is a continuous $3^{\text {rd }} t$-norm of H-type and assume that $\lim _{t \rightarrow \infty} S^{*}(x, y, z, t)=1, \quad \forall x, y, z \in X, \quad t>0 . \quad$ Let $f: X \longrightarrow X$ be a generalized Kannan-type mapping under a gauge function $\phi$ on $X$. Then, $f$ has a unique fixed point $x \in X$ and, for every $x_{0} \in X, \lim _{n} f^{n}\left(x_{0}\right)=x$.

Proof. Choose $x_{0} \in X$ and let $x_{n}=f x_{n-1}$ be the iterative sequence generated by $x_{0}$. We will show that $\left\{x_{n}\right\}$ is a
Cauchy sequence. Indeed, if not so, by definition, there exists $0<\varepsilon<1$ for which we can find $t>0$ and subsequences $\left\{x_{m(k)}\right\}$ and $\left\{x_{n(k)}\right\}$ of $\left\{x_{n}\right\}$ with $n(k)>m(k)>k$ for all positive $k$ such that

$$
S^{*}\left(x_{m(k)}, x_{m(k)}, x_{n(k)}, \phi(t)\right) \leq 1-\varepsilon, \quad t>0 .
$$

So, for $\forall t, t_{1}, t_{2}>0$ with $t=t_{1}+t_{2}$ and $a, b>0$ with $0<a+b<1$, we have

$$
\begin{aligned}
1-\varepsilon & \geq S^{*}\left(x_{m(k)}, x_{m(k)}, x_{n(k)}, \phi(t)\right) \\
& =S^{*}\left(f x_{m(k)-1}, f x_{m(k)-1}, f x_{n(k)-1}, \phi(t)\right) \\
& \geq \psi\left(S^{*}\left(x_{m(k)-1}, x_{m(k)-1}, f x_{m(k)-1}, \frac{t_{1}}{a}\right), S^{*}\left(x_{n(k)-1}, x_{n(k)-1}, f x_{n(k)-1}, \frac{t_{2}}{b}\right)\right) \\
& =\psi\left(S^{*}\left(x_{m(k)-1}, x_{m(k)-1}, x_{m(k)}, \frac{t_{1}}{a}\right), S^{*}\left(x_{n(k)-1}, x_{n(k)-1}, x_{n(k)}, \frac{t_{2}}{b}\right)\right) .
\end{aligned}
$$

Therefore,

$1-\varepsilon \geq \psi\left(S^{*}\left(x_{m(k)-1}, x_{m(k)-1}, x_{m(k)}, \frac{t_{1}}{a}\right), S^{*}\left(x_{n(k)-1}, x_{n(k)-1}, x_{n(k)}, \frac{t_{2}}{b}\right)\right)$.

By Lemma 9, for $\forall t>0$, we get

$$
\lim _{n \longrightarrow \infty} S^{*}\left(x_{n+1}, x_{n+1}, x_{n}, \phi(t)\right)=1 \text {. }
$$

So we can choose $k$ large enough such that

$$
\begin{gathered}
S^{*}\left(x_{m(k)-1}, x_{m(k)-1}, x_{m(k)}, \frac{t_{1}}{a}\right)>1-\varepsilon, \\
S^{*}\left(x_{n(k)-1}, x_{n(k)-1}, x_{n(k)}, \frac{t_{2}}{b}\right)>1-\varepsilon .
\end{gathered}
$$

Therefore, from (62) and (64) and the definition of $\Psi$-function, it follows that

$$
1-\varepsilon \geq \psi(1-\varepsilon, 1-\varepsilon)>1-\varepsilon,
$$

which is a contradiction. So $\left\{x_{n}\right\}$ is a Cauchy sequence. The completeness of $S^{*}$-fuzzy metric space $\left(X, S^{*}, *\right)$ implies that $\lim _{n \rightarrow \infty} x_{n}=x$ for some $x \in X$. Now, we claim that $f x=x$. If possible, let $S^{*}(x, x, f x, \phi(t))<1$, for some $t>0$.

Since $0<b<1$, we have chosen $\eta_{1}, \eta_{2}, t_{1}, t_{2}>0$ such that

$$
\begin{aligned}
\phi(t) & =\eta_{1}+\eta_{2}+\phi\left(t_{1}+t_{2}\right), \\
\frac{t_{2}}{b}>\phi(t) . &
\end{aligned}
$$

Then, we have

$$
\begin{aligned}
& S^{*}(x, x, f x, \phi(t)) \\
& \geq *\left(S^{*}\left(x, x, x_{n}, \eta_{1}\right), S^{*}\left(x, x, x_{n}, \eta_{2}\right), S^{*}\left(f x, f x, x_{n}, \phi\left(t_{1}+t_{2}\right)\right)\right) \\
& \quad=*\left(S^{*}\left(x, x, x_{n}, \eta_{1}\right), S^{*}\left(x, x, x_{n}, \eta_{2}\right), S^{*}\left(x_{n}, x_{n}, f x, \phi\left(t_{1}+t_{2}\right)\right)\right) \\
& \quad \geq *\left(S^{*}\left(x, x, x_{n}, \eta_{1}\right), S^{*}\left(x, x, x_{n}, \eta_{2}\right), \psi\left(S^{*}\left(x_{n}, x_{n}, x_{n+1}, \frac{t_{1}}{a}\right), S^{*}\left(x_{n-1}, x_{n-1}, x_{n}, \frac{t_{2}}{b}\right)\right)\right) \\
& \quad \geq *\left(S^{*}\left(x, x, x_{n}, \eta_{1}\right), S^{*}\left(x, x, x_{n}, \eta_{2}\right), \psi\left(S^{*}\left(x_{n}, x_{n}, x_{n+1}, \frac{t_{1}}{a}\right), S^{*}\left(x_{n-1}, x_{n-1}, x_{n}, \phi(t)\right)\right)\right) . \\
& \text { and the convergence of }\left\{x_{n}\right\} \text { to } x, \text { there } \quad S^{*}\left(x, x, x_{n}, \eta_{2}\right)>S^{*}(x, x, f x, \phi(t)), \\
& \text { integer } N_{1} \text { such that, for } \forall n>N_{1}, \\
& \left.\quad S_{n}, x_{n}, x_{n+1}, \frac{t_{1}}{a}\right)>S^{*}(x, x, f x, \phi(t)) .
\end{aligned}
$$

By Lemma 9 and the convergence of $\left\{x_{n}\right\}$ to $x$, there exists a positive integer $N_{1}$ such that, for $\forall n>N_{1}$, 
Thus, it follows that

$$
S^{*}(x, x, f x, \phi(t))>S^{*}(x, x, f x, \phi(t)),
$$

which is a contradiction. Hence, $S^{*}(x, x, f x, t)=1, \forall t>0$; therefore, $x$ is a fixed point of $f$.

In order to prove the uniqueness, suppose that $x$ and $y$ are two fixed points of $f$. Therefore, with all of the above assumptions on $a, b, t_{1}, t_{2}$, for $\forall t>0$, we have

$$
\begin{aligned}
S^{*}(x, x, y, \phi(t)) & =S^{*}(f x, f x, f y, \phi(t)) \\
& \geq \psi\left(S^{*}\left(x, x, f x, \frac{t_{1}}{a}\right), S^{*}\left(y, y, f y, \frac{t_{2}}{b}\right)\right) \\
& =\psi\left(S^{*}\left(x, x, x, \frac{t_{1}}{a}\right), S^{*}\left(y, y, f y, \frac{t_{2}}{b}\right)\right) \\
& =\psi(1,1) \\
& =1 .
\end{aligned}
$$

Thus, $x=y$, completing the proof.

Next, we prove the common fixed point theorems for weakly compatible mappings along with (E.A.) property and $\left(C L R_{g}\right)$ property.

Definition 15. Let $f$ and $g$ be two self-mappings of an $S^{*}$-fuzzy metric space $\left(X, S^{*}, *\right)$. The mappings are said to be weakly compatible, if they commute at their coincidence points; that is, $f x=g x$ implies $f g x=g f x$.

Definition 16. Let $f$ and $g$ be two self-mappings of an $S^{*}$-fuzzy metric space $\left(X, S^{*}, *\right)$. We say that $f$ and $g$ satisfy the (E.A.) property if there exists a sequence $\left\{x_{n}\right\}$ such that $\lim _{n \longrightarrow \infty} f x_{n}=\lim _{n \longrightarrow \infty} g x_{n}=x$, for some $x \in X$.

Remark 4. Note that the weakly compatible and (E.A.) property are independent to each other as is follows from the following examples.

Example 6. Let $\left(X, S^{*}, *\right)$ be an $S^{*}$-fuzzy metric space, where $X=[0,2]$ and $S^{*}$ is defined by $S^{*}(x, y, z, t)=(t /(t+|x-z|+|y-z|)), \quad \forall x, y, z \in X$ and $t>0$. $*$ is defined by $*(a, b, c)=a \cdot b \cdot c, \forall a, b, c \in[0,1]$.

Define $f, g: X \longrightarrow X$ by

$$
\begin{aligned}
& f x=\left\{\begin{array}{l}
2-x, \quad x \in[0,1], \\
0, \quad x \in(1,2],
\end{array}\right. \\
& \operatorname{gx} \begin{cases}1, & x \in[0,1], \\
\frac{3}{2}, & x \in(1,2] .\end{cases}
\end{aligned}
$$

Then, for the sequence $\left\{x_{n}\right\}=\{1-(1 / n)\}, n \geq 2$, we have $\lim _{n \longrightarrow \infty} f\left(1-\frac{1}{n}\right)=\lim _{n \longrightarrow \infty}\left(1+\frac{1}{n}\right)=\lim _{n \longrightarrow \infty} g\left(1-\frac{1}{n}\right)=1 \in[0,2]$.

Thus, $f$ and $g$ satisfy (E.A.) property.
Furthermore, $f$ and $g$ are weakly compatible, since $x=1$ is their coincidence point and $f(1)=g(1)=1=f g(1)=$ $g f(1)$.

Example 7. Let $\left(X, S^{*}, *\right)$ be an $S^{*}$-fuzzy metric space, where $X=\mathbb{R}^{+}, *(a, b, c)=a \cdot b \cdot c, \forall a, b, c \in[0,1]$, and $S^{*}(x, y, z, t)=t /(t+|x-z|+|y-z|+|x-y|), \quad \forall x, y, z \in$ $X$ and $t>0$.

Define $f, g: X \longrightarrow X$ by

$$
f x= \begin{cases}0, & 0<x \leq 1, \\ 1, & x>1, \quad x=0,\end{cases}
$$

$g x=[x]$ (the integer part of $x) \forall x \in X$.

Consider the sequence $\left\{x_{n}\right\}=\{1+(1 / n)\}, n \geq 2$. Then, we have

$$
\lim _{n \rightarrow \infty} f x_{n}=1=\lim _{n \longrightarrow \infty} g x_{n} .
$$

At the same time, for the sequence $\left\{y_{n}\right\}=\{1-(1 / n)\}$, $n \geq 2$, one has

$$
\lim _{n \longrightarrow \infty} f y_{n}=0=\lim _{n \longrightarrow \infty} g y_{n} .
$$

Thus, $f$ and $g$ satisfy the (E.A.) property. However, $f$ and $g$ are not weakly compatible since each $u \in(0,1)$ and $v \in(0,1)$ are coincidence points of $f$ and $g$ while they do not commute. Moreover, they commute at $x=0,1,2, \ldots$ but none of them are coincidence points of $f$ and $g$. Hence, (E.A.) property does not imply weak compatibility.

Definition 17. Let $f$ and $g$ are two self-mappings of an $S^{*}$-fuzzy metric space $\left(X, S^{*}, *\right)$. We say that $f$ and $g$ satisfy the common limit in the range of $g\left(C L R_{g}\right)$ property, if there exists a sequence $\left\{x_{n}\right\}$ such that $\lim _{n \longrightarrow \infty} f x_{n}=\lim _{n \longrightarrow \infty}$ $g x_{n}=g x$, for some $x \in X$.

Definition 18. Let $f$ and $g$ be two self-mappings of an $S^{*}$-fuzzy metric space $\left(X, S^{*}, *\right)$. The pair of mappings $(f, g)$ is said to be generalized Kannan-type mappings under a gauge function $\phi$ if, for $\forall x, y \in X$,

$$
S^{*}(f x, f x, f y, \phi(t)) \geq \psi\left(S^{*}\left(g x, g x, f x, \frac{t_{1}}{a}\right), S^{*}\left(f y, f y, g y, \frac{t_{2}}{b}\right)\right),
$$

where $t, t_{1}, t_{2}, a, b>0$ with $t=t_{1}+t_{2}, 0<a+b<1, \phi \in \Phi_{w}$.

In what follows, we prove a result concerning weakly compatible mappings along with (E.A.) property.

Theorem 6. Let $f$ and $g$ be two self-mappings of an $S^{*}-f u z z y$ metric space $\left(X, S^{*}, *\right)$ with a continuous $3^{\text {rd }}$ t-norm of H-type. Suppose that the following conditions are satisfied:

(1) $f$ and $g$ satisfy (E.A.) property.

(2) The pair $(f, g)$ is generalized Kannan-type mappings under a gauge function $\phi$.

(3) $g(X)$ is a closed subspace of $X$.

(4) $f$ and $g$ are weakly compatible on $X$, provided $0<S^{*}(x, y, z, t)<1, \forall t>0$. 
Then, $f$ and $g$ have a unique common fixed point.

Proof. Since $f$ and $g$ satisfy (E.A.) property, there exists a sequence $\left\{x_{n}\right\}$ in $X$ such that

$$
\lim _{n \longrightarrow \infty} f x_{n}=\lim _{n \longrightarrow \infty} g x_{n}=u \in X
$$

Since $g(X)$ is a closed subspace of $X$, every convergent sequence of points of $g(X)$ has a limit in $g(X)$. Therefore, there is $a \in X$ such that

$$
u=\lim _{n \longrightarrow \infty} f x_{n}=\lim _{n \longrightarrow \infty} g x_{n}=g a \in g(X) .
$$

We now show that $f a=u=g a$.

If possible, let $0<S^{*}(g a, g a, f a, \phi(t))<1$, for some $t>0$. Since $0<b<1$, we can choose $\eta_{1}, \eta_{2}, t_{1}, t_{2}>0$ such that

$$
\begin{aligned}
\phi(t) & =\eta_{1}+\eta_{2}+\phi\left(t_{1}+t_{2}\right), \\
\frac{t_{2}}{b}>\phi(t) . &
\end{aligned}
$$

Then, we get

$$
\begin{aligned}
S^{*} & (g a, g a, f a, \phi(t)) \\
& \geq *\left(S^{*}\left(g a, g a, f x_{n}, \eta_{1}\right), S^{*}\left(g a, g a, f x_{n}, \eta_{2}\right), S^{*}\left(f a, f a, f x_{n}, \phi\left(t_{1}+t_{2}\right)\right)\right) \\
& =*\left(S^{*}\left(g a, g a, f x_{n}, \eta_{1}\right), S^{*}\left(g a, g a, f x_{n}, \eta_{2}\right), S^{*}\left(f x_{n}, f x_{n}, f a, \phi\left(t_{1}+t_{2}\right)\right)\right) \\
& \geq *\left(S^{*}\left(g a, g a, f x_{n}, \eta_{1}\right), S^{*}\left(g a, g a, f x_{n}, \eta_{2}\right), \psi\left(S^{*}\left(g x_{n}, g x_{n}, f x_{n}, \frac{t_{1}}{a}\right), S^{*}\left(f a, f a, g a, \frac{t_{2}}{b}\right)\right)\right) \\
& \geq *\left(S^{*}\left(g a, g a, f x_{n}, \eta_{1}\right), S^{*}\left(g a, g a, f x_{n}, \eta_{2}\right), \psi\left(S^{*}\left(g x_{n}, g x_{n}, f x_{n}, \frac{t_{1}}{a}\right), S^{*}(f a, f a, g a, \phi(t))\right)\right) .
\end{aligned}
$$

By (78), there exists a positive integer $N_{2}$ such that, for $\forall n>N_{2}$,

$$
\min \left\{S^{*}\left(g a, g a, f x_{n}, \eta_{1}\right), S^{*}\left(g a, g a, f x_{n}, \eta_{2}\right), S^{*}\left(g x_{n}, g x_{n}, f x_{n}, \frac{t_{1}}{a}\right)\right\}>S^{*}(g a, g a, f a,(t)) .
$$

Then, we have

$$
S^{*}(g a, g a, f a, \phi(t))>S^{*}(g a, g a, f a, \phi(t)),
$$

which is a contradiction. Hence, $S^{*}(g a, g a, f a, \phi(t))=1$, $\forall t>0$, which implies $f a=u=g a$.

Since $f$ and $g$ are weakly compatible, it follows that $g f a=f g a$; i.e., $g u=f u$.

Now, we will prove that $u$ is a common fixed point of $f$ and $g$.

From condition (2), we deduce

$$
\begin{aligned}
& S^{*}\left(f u, f u, f x_{n}, \phi(t)\right) \\
& \quad \geq \psi\left(S^{*}\left(g u, g u, f x_{n}, \frac{t_{1}}{a}\right), S^{*}\left(f x_{n}, f x_{n}, g x_{n}, \frac{t_{2}}{b}\right)\right),
\end{aligned}
$$

where $t=t_{1}+t_{2}$. Taking the limit as $n \longrightarrow \infty$, we have
$S^{*}(f u, f u, u, \phi(t))$

$$
\begin{aligned}
& \geq \psi\left(S^{*}\left(g u, g u, f u, \frac{t_{1}}{a}\right), S^{*}\left(f u, f u, g u, \frac{t_{2}}{b}\right)\right) \\
& =\psi(1,1) \\
& =1 .
\end{aligned}
$$

Hence, $f u=u=g u$; that is, $u$ is a common fixed point of $f$ and $g$.

In order to prove the uniqueness, let $u$ and $v$ be two common fixed points of $f$ and $g$. Therefore, for $\forall t_{1}, t_{2}, t, a, b>0$ with $t=t_{1}+t_{2}, 0<a+b<1$, one has $S^{*}(u, u, v, \phi(t))=S^{*}(f u, f u, f v, \phi(t))$

$$
\begin{aligned}
& \geq \psi\left(S^{*}\left(g u, g u, f u, \frac{t_{1}}{a}\right), S^{*}\left(f v, f v, g v, \frac{t_{2}}{b}\right)\right) \\
& =\psi\left(S^{*}\left(u, u, u, \frac{t_{1}}{a}\right), S^{*}\left(v, v, v, \frac{t_{2}}{b}\right)\right) \\
& =\psi(1,1) \\
& =1 .
\end{aligned}
$$


Hence, $u=v$; that is, $u$ is a unique common fixed point of $f$ and $g$.

Finally, we present a theorem for weakly compatible mappings along with $\left(C L R_{g}\right)$ property as follows.

Theorem 7. Let $f$ and $g$ be two self-mappings of an $S^{*}-f u z z y$ metric space $\left(X, S^{*}, *\right)$ with a continuous $3^{\text {rd }} t$-norm of H-type. Suppose that the following conditions hold:

(1) $f$ and $g$ satisfy $\left(C L R_{g}\right)$ property.

(2) The pair $(f, g)$ is generalized Kannan-type mappings under a gauge function $\phi$.

(3) $f$ and $g$ are weakly compatible on $X$, provided $0<S^{*}(x, y, z, t)<1, \forall t>0$.

Then, $f$ and $g$ have a unique common fixed point.
Proof. Since $f$ and $g$ satisfy the $\left(C L R_{g}\right)$ property, there exists a sequence $\left\{x_{n}\right\}$ in $X$ such that

$$
\lim _{n \longrightarrow \infty} f x_{n}=\lim _{n \longrightarrow \infty} g x_{n}=g x \in X .
$$

We will show that $f x=g x$.

If possible, let $0<S^{*}(g a, g a, f a, \phi(t))<1$, for some $t>0$. Since $0<b<1$, we can choose $\eta_{1}, \eta_{2}, t_{1}, t_{2}>0$ such that

$$
\begin{aligned}
\phi(t) & =\eta_{1}+\eta_{2}+\phi\left(t_{1}+t_{2}\right), \\
\frac{t_{2}}{b}>\phi(t) . &
\end{aligned}
$$

Then, we have

$$
\begin{aligned}
S^{*} & (f x, f x, g x, \phi(t)) \\
& \geq *\left(S^{*}\left(f x, f x, f x_{n}, \eta_{1}\right), S^{*}\left(f x, f x, f x_{n}, \eta_{2}\right), S^{*}\left(g x, g x, f x_{n}, \phi\left(t_{1}+t_{2}\right)\right)\right) \\
& =*\left(S^{*}\left(f x, f x, f x_{n}, \eta_{1}\right), S^{*}\left(f x, f x, f x_{n}, \eta_{2}\right), S^{*}\left(f x_{n}, f x_{n}, f x, \phi\left(t_{1}+t_{2}\right)\right)\right) \\
& \geq *\left(S^{*}\left(f x, f x, f x_{n}, \eta_{1}\right), S^{*}\left(f x, f x, f x_{n}, \eta_{2}\right), \psi\left(S^{*}\left(g x_{n}, g x_{n}, f x_{n}, \frac{t_{1}}{a}\right), S^{*}\left(f x, f x, g x, \frac{t_{2}}{b}\right)\right)\right) \\
& \geq *\left(S^{*}\left(f x, f x, f x_{n}, \eta_{1}\right), S^{*}\left(f x, f x, f x_{n}, \eta_{2}\right), \psi\left(S^{*}\left(g x_{n}, g x_{n}, f x_{n}, \frac{t_{1}}{a}\right), S^{*}(f x, f x, g x, \phi(t))\right)\right) .
\end{aligned}
$$

By (86), there exists a positive integer $N_{3}$ such that, for $\forall n>N_{3}$,

$$
\begin{gathered}
S^{*}\left(f x, f x, f x_{n}, \eta_{1}\right)>S^{*}(f x, f x, g x, \phi(t)), \\
S^{*}\left(f x, f x, f x_{n}, \eta_{2}\right)>S^{*}(f x, f x, g x, \phi(t)), \\
S^{*}\left(g x_{n}, g x_{n}, f x_{n}, \frac{t_{1}}{a}\right)>S^{*}(f x, f x, g x, \phi(t)) .
\end{gathered}
$$

Then, we have

$$
S^{*}(f x, f x, g x, \phi(t))>S^{*}(f x, f x, g x, \phi(t)),
$$

which is a contradiction. Therefore, $S^{*}(f x, f x, g x, \phi(t))=$ $1, \forall t>0$, which implies $f x=g x=u$.

Using the similar argument to that in the proof of Theorem 6 , we can obtain that $u$ is a unique common fixed point of $f$ and $g$.

\section{Data Availability}

No data sets were generated or analyzed during the current study.

\section{Conflicts of Interest}

The authors declare that they have no conflicts of interest.

\section{Acknowledgments}

Mi Zhou was partially supported by the Hainan Provincial Natural Science Foundation of China (Grant no.
118MS081) and Science and Technology Cooperation Project of Sanya City (Grant no. 2018YD13 and 2018YD08). Xiao-lan Liu was partially supported by the National Natural Science Foundation of China (Grant nos. 61573010 and 11872043), Sichuan Science and Technology Program (Grant no. 2019YJ0541), Scientific Research Project of Sichuan University of Science and Engineering (Grant nos. 2017RCL54 and 2019RC42), and Opening Project of Sichuan Province University Key Laboratory of Bridge Non-Destruction Detecting and Engineering Computing (Grant no. 2019QZJ03).

\section{References}

[1] L. A. Zadeh, "Fuzzy sets," Information and Control, vol. 8, no. 3, pp. 338-353, 1965

[2] I. Kramosil and J. Michálek, "Fuzzy metrics and statistical metric spaces," Kybernetika, vol. 11, no. 5, pp. 336-344, 1975.

[3] A. George and P. Veeramani, "On some results in fuzzy metric spaces," Fuzzy Sets and Systems, vol. 64, no. 3, pp. 395-399, 1994.

[4] O. Kaleva and S. Seikkala, "On fuzzy metric spaces," Fuzzy Sets and Systems, vol. 12, no. 3, pp. 215-229, 1984.

[5] S. Kumar, "Common fixed point theorem in fuzzy 2-metric spaces," Scientific Studies and Research Series Mathematics and Informatics, vol. 18, pp. 111-116, 2008.

[6] S. Sharama, "On fuzzy metric space," Southeast Asian Bulletin of Mathematics, vol. 26, pp. 133-145, 2002.

[7] B. Singh and M. S. Chauhan, "Generalized fuzzy metric spaces and fixed point theorems," Bulletin of the Calcutta Mathematical Society, vol. 89, pp. 457-460, 1997. 
[8] G. Sun and K. Yang, "Generalized fuzzy metric spaces with properties," Research Journal of Applied Science, Engineering and Technology, vol. 2, no. 7, pp. 673-678, 2010.

[9] S. Sedghi and N. Shobe, "Fixed point theorems in $M$-fuzzy metric with property (E)." Advances in Fuzzy Mathematics, vol. 1, no. 1, pp. 55-65, 2006.

[10] S. Sedghi, N. Shobe, and A. Aliouche, "A generalization of fixed point theorems in $S$-metric spaces," Matematički Vesnik, vol. 64, no. 3, pp. 258-266, 2012.

[11] R. Kannan, "Some results on fixed point," Bulletin of the Calcutta Mathematical Society, vol. 60, pp. 71-76, 1968.

[12] P. V. Subrahmanyam, "Completeness and fixed-points," Monatshefte für Mathematik, vol. 80, no. 4, pp. 325-330, 1975.

[13] B. S. Choudhury and K. Das, "A coincidence point result in Menger spaces using a control function," Chaos, Solitons \& Fractals, vol. 42, no. 5, pp. 3058-3063, 2009.

[14] J. X. Fang, "Fixed point theorems of local contraction mappings on Menger spaces," Applied Mathematics and Mechanics, vol. 12, pp. 363-372, 1991.

[15] D. O'Regan and R. Saadati, "Nonlinear contraction theorems in probabilistic spaces," Applied Mathematics and Computation, vol. 195, pp. 86-93, 2008.

[16] L. Ćirić, "Sovling the Banach fixed point principle for nonlinear contractions in probabilistic metric spaces," Nonlinear Analysis, vol. 72, pp. 2009-2018, 2010.

[17] J.-X. Fang, "On $\varphi$-contractions in probabilistic and fuzzy metric spaces," Fuzzy Sets and Systems, vol. 267, pp. 86-99, 2015.

[18] J. Jachymski, "On probabilistic $\varphi$-contraction on Menger spaces,” Nonlinear Analysis, vol. 73, pp. 1131-1137, 2010.

[19] A. Francisco, R. L. Hierroa, and W. Sintunavarat, "Common fixed point theorems in fuzzy metric spaces using the CLR property," Fuzzy Sets and Systems, vol. 282, pp. 131-142, 2014.

[20] Y. Shi, L. Ren, and X. Wang, "The extension of fixed point theorems for set valued mapping," Journal of Applied Mathematics and Computing, vol. 13, no. 1-2, pp. 277-286, 2003.

[21] O. Hadžić, "A fixed point theorem in Menger spaces," Publications de l'Institut Mathématique, vol. 20, pp. 107-112, 1979. 